This is the final peer-reviewed accepted manuscript of:

Muratov, C. B., Novaga, M., \& Ruffini, B. (2018). On equilibrium shape of charged flat drops. Communications on Pure and Applied Mathematics, 71(6), 1049-1073.

The final published version is available online at: http://dx.doi.org/10.1002/cpa.21739

Rights / License:

The terms and conditions for the reuse of this version of the manuscript are specified in the publishing policy. For all terms of use and more information see the publisher's website. 


\title{
On Equilibrium Shape of Charged Flat Drops
}

\author{
CYRILL B. MURATOV \\ New Jersey Institute of Technology \\ MATTEO NOVAGA \\ University of Pisa \\ AND \\ BERARDO RUFFINI \\ Institut Montpelliérain Alexander Grothendieck
}

\begin{abstract}
The equilibrium shapes of two-dimensional charged, perfectly conducting liquid drops are governed by a geometric variational problem that involves a perimeter term modeling line tension and a capacitary term modeling Coulombic repulsion. Here we give a complete explicit solution to this variational problem. Namely, we show that at fixed total charge a ball of a particular radius is the unique global minimizer among all sufficiently regular sets in the $\mathrm{p}$ lane. For sets whose area is also fixed, we show that balls a re the only minimizers if the charge is less than or equal to a critical charge, while for larger charge minimizers do not exist. Analogous results hold for drops whose potential, rather than charge, is fixed. (C) 2017 Wiley Periodicals, Inc.
\end{abstract}

\section{Introduction}

In this paper we are interested in a geometric variational problem that arises in the studies of electrified liquids (for a recent o verview, s ee, e.g., [22]). Consider an insulating fluid between two parallel plates, also made from an insulating material. From a small opening in one of the plates an immiscible, electrically conducting incompressible liquid is introduced and is allowed to fill a region whose lateral dimensions greatly exceed the distance between the plates. Because of its essentially two-dimensional character, we call the resulting pancake-shaped drop of the conducting liquid a flat drop. A voltage is then applied through the opening, imparting an electric charge to the liquid. One would like to understand the equilibrium shape attained by the drop, either at constant voltage or constant total charge, and either at constant or variable volume. Physically, this problem is interesting because the electric field produced by the charges tends to destabilize the liquid interface [22, 24, 39, 51, 53], an effect that is used extensively in a variety of applications such as mass spectrometry [30], electrospinning [6], and microfluidics [15]. 
Mathematically, this problem is interesting because the interplay between the capillary and Coulombic forces produces a competition that makes prediction of the energy-minimizing configurations a highly nontrivial task.

The equilibrium configurations of electrified conducting liquids may be studied with the help of a basic variational model that goes back all the way to Lord Rayleigh [48]. Let $K \subset \mathbb{R}^{3}$ be a compact set with smooth boundary. If $\sigma$ is the surface tension, $\epsilon$ is the relative permittivity of the surrounding medium, and $\epsilon_{0}$ is the permittivity of vacuum, then the energy of a charged, perfectly conducting liquid drop occupying $K$, with volume $V$ and the total charge $Q$, is given by [39]

$$
E_{3 d}^{Q}(K)=\sigma \mathcal{H}^{2}(\partial K)+\frac{Q^{2}}{2 C(K)}
$$

where $\mathcal{H}^{s}$ denotes the $s$-dimensional Hausdorff measure, with $\mathcal{H}^{2}(\partial K)$ representing the surface area of the liquid, and

$$
\frac{1}{C(K)}:=\frac{1}{4 \pi \epsilon \epsilon_{0}} \inf _{\mu(K)=1} \iint_{K} \frac{d \mu(x) d \mu(y)}{|x-y|}
$$

is the inverse electric capacitance of $K$ in the SI units. Here $\mu$ is a probability measure supported on $K$, and the infimum in (1.2) is attained by a unique measure that concentrates on $\partial K$ [40]. If instead the voltage $U$ on the drop is prescribed, then the total energy (including that of the charge reservoir) is

$$
E_{3 d}^{U}(K):=\sigma \mathcal{H}^{2}(\partial K)-\frac{1}{2} C(K) U^{2} .
$$

For flat drops, this model can be reformulated as follows. Let $d$ be the distance between the plates and assume that $K=\Omega \times[0, d]$, where $\Omega \subset \mathbb{R}^{2}$ is a compact set with smooth boundary that represents the shape of the flat drop. Note that this means that we are assuming the contact angle $\theta=\frac{\pi}{2}$, corresponding to a flat meniscus. For a general contact angle $\theta \in[0, \pi]$ the set $K$ may be similarly defined in terms of $\Omega$, with the shape of the meniscus determined by balancing the capillary forces locally. In this case the meniscus cross section will have the shape of a circular arc of length $\ell=\frac{\pi-2 \theta}{2 \cos \theta} d$, with $\ell=d$ if $\theta=\frac{\pi}{2}$. Then, to the leading order in $d \ll L$, where $L$ is the characteristic dimension of the drop, the energy $E_{3 d}^{Q}(K)$ is given by

$$
E_{2 d}^{Q}(\Omega):=\sigma \ell \mathcal{H}^{1}(\partial \Omega)+\frac{Q^{2}}{8 \pi \epsilon \epsilon_{0}} \mathcal{I}_{1}(\Omega)
$$

where we introduced the Riesz capacitary energy of a set:

$$
\mathcal{I}_{\alpha}(\Omega):=\inf \left\{\iint_{\Omega} \frac{d \mu(x) d \mu(y)}{|x-y|^{\alpha}}: \mu(\Omega)=1\right\},
$$


which is more generally defined for all Borel sets $\Omega \subset \mathbb{R}^{N}$ and $\alpha \in(0, N)$. Similarly, $E_{3 d}^{U}(K)$ becomes

$$
E_{2 d}^{U}(\Omega):=\sigma \ell \mathcal{H}^{1}(\partial \Omega)-\frac{2 \pi \epsilon \epsilon_{0} U^{2}}{\mathcal{I}_{1}(\Omega)} .
$$

The volume of the flat drop is, to the leading order, equal to $V=|\Omega| d$, where $|\Omega|$ denotes the area of $\Omega$. Hence, fixing the volume of $K$ amounts to fixing $|\Omega|$.

We note that the infimum in the definition of $\mathcal{I}_{1}(\Omega)$ is attained by a unique measure supported on $\Omega$, and the value of $\mathcal{I}_{1}(\Omega)$ is proportional to the inverse of the Riesz capacity of $\Omega$ [40]. Also, for fixed volume there is no shape-dependent contribution to the energy from the liquid-solid interface to the leading order. We point out that the same type of mathematical models is relevant to systems that give rise to electric charges concentrating on planar regions, such as high- $T_{c}$ superconductors [21], Langmuir monolayers [5], and possibly graphene [49]. In addition, it should be possible to rigorously obtain the corresponding two-dimensional expressions for the energy from the original three-dimensional energy in the limit $d \rightarrow 0$ via $\Gamma$-convergence for a suitable notion of convergence of sets [11].

We are now in a position to introduce the dimensionless versions of the energies that we will analyze. Setting

$$
\mathcal{E}_{\lambda}^{Q}(\Omega):=\frac{E_{2 d}^{Q}(L \Omega)}{\sigma \ell L}, \quad \lambda:=\frac{Q^{2}}{8 \pi \epsilon \epsilon_{0} \sigma \ell L^{2}},
$$

we obtain a one-parameter family of energy functionals

$$
\mathcal{E}_{\lambda}^{Q}(\Omega)=\mathcal{H}^{1}(\partial \Omega)+\lambda \mathcal{I}_{1}(\Omega),
$$

with $\lambda>0$ a fixed dimensionless parameter, defined on all compact subsets of $\mathbb{R}^{2}$. Similarly, setting

$$
\mathcal{E}_{\lambda}^{U}(\Omega):=\frac{E_{2 d}^{U}(L \Omega)}{\sigma \ell L}, \quad \lambda:=\frac{2 \pi \epsilon \epsilon_{0} U^{2}}{\sigma \ell},
$$

we obtain a one-parameter family of energy functionals,

$$
\mathcal{E}_{\lambda}^{U}(\Omega)=\mathcal{H}^{1}(\partial \Omega)-\frac{\lambda}{\mathcal{I}_{1}(\Omega)},
$$

with $\lambda>0$ a fixed dimensionless parameter. These energy functionals and their global minimizers with or without constraints are the main subject of the present paper.

There has recently been great interest in nonlocal variational problems involving energies like those in (1.8) and (1.10). In particular, the nonlocal isoperimetric problems governed by (1.1) or (1.8), or their generalizations involving Riesz energy, in which the measure $\mu$ is fixed to be the uniform measure, was recently treated in [9, 23, 25, 26, 35- -37, 41, 44] (see also [2, 16, 31, 32, 38] for closely related problems on large bounded domains; these lists are not at all meant to be exhaustive). One of the motivations for those studies comes from the fact that the 
three-dimensional problem with uniform charge density corresponds to the famous model of the atomic nucleus introduced by Gamow [27] and is used to explain the stability of nuclear matter and nuclear fission [8, 52]. It is now known that in the case of the energy in $(1.8)$ with $\mathcal{I}_{1}$ evaluated, with $d \mu=|\Omega|^{-1} d x$, the unique minimizer with $|\Omega|=m$ fixed is a ball as long as $m \leq m_{0} \lambda^{-1}$, where $m_{0}>0$ is a universal constant. On the other hand, there is no minimizer with $|\Omega|=m$ as soon as $m>m_{2} \lambda^{-1}$, where $m_{2} \geq m_{0}$ is another universal constant [36].

These conclusions have been extended to the case of higher-dimensional problems and more general Riesz kernels [23, 35, 37, 41]. The basic intuition behind these results is that when the value of $m$ is sufficiently small, the perimeter term dominates the energy, forcing the minimizer to coincide with the one for the classical isoperimetric problem. In contrast, when the value of $m$ is sufficiently large, the repulsive term becomes dominant, forcing the charges to move off to infinity. The observations above motivate the conjecture, first stated in [17], that minimizers of the problem with uniform charge density are balls unless it is better to split one ball into to two equal-size balls. Whether this conjecture is true in its full generality is a very difficult question, but it was answered in the affirmative in the case of two-dimensional problems involving Riesz kernels with a sufficiently small exponent $\alpha$ [36, 44]. A weaker version of this result in higher dimensions, namely, that minimizers of the above problem with $\alpha$ sufficiently small are balls whenever they exist, without specifying the threshold for existence, was established in [9].

One might expect that the capacitary problem in (1.1) should exhibit a similar behavior. Studies of the instability of balls with respect to small smooth perturbations in three space dimensions go back to Lord Rayleigh [48], and emergence of regular nonsymmetric critical points out of the bifurcation at the critical charge has been established much more recently in [24] (for some numerical work, see also [7, 13, 29]). Yet a striking result from a recent paper [33] shows that the problem of minimizing the energy in (1.1) never admits global minimizers with $|\Omega|=m$. Even worse, this problem was shown never to admit any regular local minimizers, understood in any reasonable sense [43]. This severe illposedness of the three-dimensional capacitary problem has to do with an incompatibility between the perimeter term, which sees two-dimensional sets, and the Coulombic term, which sees sets of positive Newtonian capacity. The latter, however, could concentrate on sets of positive $s$-dimensional Hausdorff measure with $1<s<2$ [40]. Thus, there is a gap between the Hausdorff dimension of the sets that are negligible with respect to the perimeter and those negligible with respect to the Coulombic energy, which prevents the perimeter from imparting classical regularity to the minimizers. Note that existence may be restored if one looks for minimizers in more regular classes, such as, for example, the class of convex compact sets [34]. Also, well-posedness under confinement in three dimensions can be restored by including entropic effects into the model, resulting in a finite screening length [43]. 
Coming back to our main problem of interest, which consists of minimizing $\mathcal{E}_{\lambda}^{Q}(\Omega)$ in (1.8) among all compact sets $\Omega \subset \mathbb{R}^{2}$ with $|\Omega|=m$ fixed, one can see that the reduced dimensionality of the problem makes it a borderline case between the ill-posed capacitary problem in three-dimensions and the well-posed uniform charge density problem in two dimensions. Indeed, in this case the perimeter controls sets of zero Riesz capacity for $\alpha=1$ in two space dimensions. Therefore, the question of existence vs. nonexistence of minimizers in this setting becomes particularly delicate. In what follows, we will show that the nonlocal isoperimetric problem associated with (1.8) remains on the "good side" of the critical value of $\alpha=1$, exhibiting existence of minimizers for small values of $m$ and nonexistence for large values of $m$.

Remarkably, we are able to give a complete explicit solution to this problem, both with and without the constraint on $|\Omega|$. In the absence of the constraint, the energy $\mathcal{E}_{\lambda}^{Q}$ is uniquely minimized by balls of radius $R=\frac{1}{2} \sqrt{\lambda}$. With the constraint $|\Omega|=m$ the unique minimizers of $E_{\lambda}^{Q}$ are balls when $\lambda \leq \frac{4 m}{\pi}$, while there are no minimizers when $\lambda>\frac{4 m}{\pi}$. We note that balls lose their minimizing property before it becomes energetically favorable to split one ball into two well-separated balls with equal area and charge, which happens for $\lambda>4 m \sqrt{2} / \pi$, or before deforming a ball into an elliptical domain of the same area lowers energy, which happens for $\lambda>\frac{12 m}{\pi}$. The nature of the instability for $\lambda>\frac{4 m}{\pi}$ is similar to the one for the three-dimensional capacitary problem [33]: one can lower the energy by splitting off a large number of small balls, putting the right amount of charge on them and sending them off to infinity. This catastrophic loss of stability suggests that the ill-posedness exhibited by the three-dimensional problem [33 43] begins to manifest itself in our two-dimensional problem at a critical value of charge. Lastly, we also provide a complete solution to the problem of minimizing $\mathcal{E}_{\lambda}^{U}$ from (1.10). Without the constraint on $|\Omega|$, this energy has minimizers if and only if $\lambda=\pi^{2}$, and the only minimizers are balls (of any radius). If also $|\Omega|=m$, then balls of area $m$ are the only minimizers when $\lambda \leq \pi^{2}$, and there are no minimizers for $\lambda>\pi^{2}$.

The strategy of the proof of our main theorems is to take advantage of the fact that without the volume constraint the energy $\mathcal{E}_{\lambda}^{Q}$ of a set $\Omega$ decreases if we convexify each connected component of $\Omega$. Then we exploit the fact that the perimeter is linear for the Minkowski sum [28], while the nonlocal term $\mathcal{I}_{1}$ is sublinear [45]. This, together with some classical, simple results in the theory of convex bodies allows us to transform each convex component of a set into a ball. Eventually we show that by suitably merging several balls into one ball we decrease the energy. Let us stress that such a technique is very special and applies only to the two-dimensional case and only to the capacitary energy $\mathcal{I}_{1}$. It is indeed an open 
problem whether $\mathcal{I}_{\alpha}$ is a subadditive operator with respect to the Minkowski summation for $\alpha \neq 1$ (and for $\alpha \neq N-2$ in $\mathbb{R}^{N}$ with $N \geq 3$ ). For the constrained problem, we combine the previous result with the well-known maximizing property of balls with respect to the Riesz capacitary energy [46, 47] to prove existence for small charges. Finally, the nonexistence for large charges for the constrained problem is obtained by constructing a test configuration with many balls whose energy in the limit coincides with that of the unconstrained problem.

Let us also point out that while our paper is concerned exclusively with global energy minimizers, existence of local minimizers or critical points of the energy is naturally an interesting question, both physically and mathematically. Care, however, is needed in defining the appropriate notion of solution in view of the a priori lack of regularity and potentially severe ill-posedness of the considered variational problems [43]. In particular, one needs to take into consideration the possibility of either local minimizers or critical points consisting of sets with many small holes ("froth"). Some new quantitative insights into the capacitary energy of sets of finite perimeter would be needed to deal with those issues, which goes beyond the scope of the present work.

The rest of the paper is organized as follows: in Section 2, we give the precise definitions of the variational problems to be studied and state our main results, in Section 3 we discuss some preliminary results, and in Section 4 we present the proofs of the main theorems.

\section{Setting and Statements of the Main Results}

In this section we state the main results of our paper. Viewing $\mathcal{E}_{\lambda}^{Q}$ in (1.8) as a map from $\mathcal{B}\left(\mathbb{R}^{2}\right)$ to $[0,+\infty]$, where $\mathcal{B}\left(\mathbb{R}^{2}\right)$ is the family of all Borel subsets of $\mathbb{R}^{2}$, we start by defining the appropriate admissible classes in the domain of $\mathcal{E}_{\lambda}^{Q}$. Given two sets $A, B \subset \mathbb{R}^{2}$ we introduce the equivalence relation $A \sim B$ if $\mathcal{H}^{1}(A \backslash B)=\mathcal{H}^{1}(B \backslash A)=0$. We then set

$$
\mathcal{A}:=\left\{\Omega \subset \mathbb{R}^{2}: \Omega \text { compact, }|\Omega|>0, \mathcal{H}^{1}(\partial \Omega)<\infty\right\} / \sim .
$$

Notice that the equivalence relation $\sim$ in the definition of class $\mathcal{A}$ will be needed in order to have uniqueness of minimizers of $\mathcal{E}_{\lambda}^{Q}$ over $\mathcal{A}$.

The definition above ensures that $0<\mathcal{E}_{\lambda}^{Q}(\Omega)<\infty$ whenever $\Omega \in \mathcal{A}$. We note that physically class $\mathcal{A}$ corresponds to flat drops with fixed charge and variable volume, which can be achieved by placing a porous membrane, permeable to the liquid but impermeable to the charge-carrying ions, into the opening supplying the liquid. Similarly, for $m>0$ we define

$$
\mathcal{A}_{m}:=\{\Omega \in \mathcal{A}:|\Omega|=m\} .
$$

Note that the definitions of both the energy and the associated admissible classes $\mathcal{A}$ and $\mathcal{A}_{m}$ involve the Hausdorff measure $\mathcal{H}^{1}$ of the topological boundary. Unfortunately, $\mathcal{H}^{1}$ fails to be lower-semicontinuous even with respect to Hausdorff 
convergence of compact sets, making it difficult to apply the direct method of the calculus of variations to establish the existence of minimizers of $\mathcal{E}_{\lambda}^{Q}$. One possibility to proceed might be to replace $\mathcal{H}^{1}(\partial \Omega)$ with $P(\Omega)$, where

$$
P(\Omega):=\sup \left\{\int_{\Omega} \nabla \cdot \phi d x: \phi \in C_{c}^{\infty}\left(\mathbb{R}^{2}, \mathbb{R}^{2}\right),\|\phi\|_{L^{\infty}\left(\mathbb{R}^{2}\right)} \leq 1\right\}
$$

is the perimeter functional (which coincides with $\mathcal{H}^{1}(\partial \Omega)$ for sets with smooth boundary [4]), and work in the class of sets of finite perimeter. This, however, would bring us to an ill-posed problem: since $\mathcal{I}_{1}$ is a capacitary term, it is not well-defined on sets that are defined up to sets of Lebesgue measure zero [40]. This means, for example, that it is possible to find a compact set consisting of a disjoint union of a closed ball of area $m$ and a closed set of Lebesgue measure zero but positive Riesz capacity (by [40, theorem 3.13], for the latter one can take any compact set $K \subset \mathbb{R}^{2}$ such that $0<\mathcal{H}^{s}(K)<\infty$ for some $1<s<2$ ). Putting all the charge on the latter and then translating and dilating it off to infinity, one recovers the perimeter of the ball of area $m$ as the infimum of the energy. Thus the infimum would clearly not be attained, and this is the reason we restrict ourselves to the classes $\mathcal{A}$ and $\mathcal{A}_{m}$.

Notice that, for all $\Omega \in \mathcal{A}$, we have $P(\Omega) \leq \mathcal{H}^{1}(\partial \Omega)$. Indeed, following [4] we have that $P(\Omega)=\mathcal{H}^{1}\left(\partial^{M} \Omega\right)$ where

$$
\partial^{M} \Omega:=\left\{x \in \mathbb{R}^{2}: \lim _{r \rightarrow 0} \frac{\left|\Omega \cap B_{r}(x)\right|}{\pi r^{2}} \notin\{0,1\}\right\} \subseteq \partial \Omega .
$$

However, $P(\Omega)=\mathcal{H}^{1}(\partial \Omega)$ as soon as $\Omega$ has Lipschitz boundary.

In this paper we do not rely on the direct method of the calculus of variations. Instead, we directly show that balls are minimizers for our problem. We also point out that by the results of [33], the infimum of the energy over $\mathcal{A}_{m}$ is never attained if $\mathcal{I}_{1}$ is replaced by $\mathcal{I}_{\alpha}$ with $\alpha \in(0,1)$, contrary to the case of $\alpha=1$ considered here.

We now state our first result concerning minimizers of $\mathcal{E}_{\lambda}^{Q}$ over $\mathcal{A}$.

THEOREM 2.1. Let $\lambda>0$ and let $R_{\lambda}=\frac{1}{2} \sqrt{\lambda}$. Then the closed ball $\bar{B}_{R_{\lambda}}$ is the unique (up to translations) minimizer of $\mathcal{E}_{\lambda}^{Q}$ over $\mathcal{A}$.

We note that the optimal radius $R_{\lambda}$ can be determined by minimizing over balls, for which the energy is explicitly (see Lemma 3.3)

$$
\mathcal{E}_{\lambda}^{Q}\left(\bar{B}_{R}\right)=2 \pi R+\frac{\lambda \pi}{2 R} \geq 2 \pi \sqrt{\lambda}=\mathcal{E}_{\lambda}^{Q}\left(\bar{B}_{R_{\lambda}}\right),
$$

with the lower bound in the right-hand side attained only if $R=R_{\lambda}$. We also note that because of the characterization of sets of finite perimeter in the plane [3], the statement of Theorem 2.1 remains true when the minimization is carried out among all sets of finite perimeter, provided that the set $\Omega$ is identified with its measuretheoretic closure. Alternatively, the statement holds when minimizing $\mathcal{E}_{\lambda}^{Q}$ among 
convex compact sets. We caution the reader, however, that not every minimizing sequence for $\operatorname{vph} \mathcal{E}_{\lambda}^{Q}$ (after suitable translations) has a ball of radius $R_{\lambda}$ as the limit.

Remark 2.2. For $\lambda>0$ the infimum of $\mathcal{E}_{\lambda}^{Q}$ over $\mathcal{A}$ is achieved by a sequence of $N$ balls of radius $R_{\lambda} / N$ running off to infinity. There are also minimizing sequences consisting of $N$ balls with $N \rightarrow \infty$. Indeed, taking $\Omega_{\varepsilon}=\bigcup_{i=1}^{N} \bar{B}_{R_{\lambda} / N}\left(x_{i}\right)$, with $\left|x_{i}-x_{j}\right| \gg R_{\lambda}$, in view of Theorem 2.1 and Lemma3.2 we have

$$
\mathcal{E}_{\lambda}^{Q}\left(\Omega_{\varepsilon}\right) \leq N \mathcal{E}_{\lambda / N^{2}}^{Q}\left(\bar{B}_{R_{\lambda} / N}\right)+\varepsilon=\mathcal{E}_{\lambda}^{Q}\left(\bar{B}_{R_{\lambda}}\right)+\varepsilon=\inf _{\Omega \in \mathcal{A}} \mathcal{E}_{\lambda}^{Q}(\Omega)+\varepsilon
$$

for an arbitrary $\varepsilon>0$. Thus, the energy can be equally minimized by a fine "mist" of droplets moving off to infinity by distributing the charge proportionally to their perimeter.

As a by-product of the proof of Theorem 2.1 we can show that balls minimize $\mathcal{I}_{1}$ (that is, maximize the capacity) among sets of fixed perimeter, thus generalizing a result in [45, cor. 3.2].

COROLlARY 2.3. The closed ball $\bar{B}_{R}$ is the unique (up to translations) minimizer of $\mathcal{I}_{1}$ among the sets $\Omega \in \mathcal{A}$ with $P(\Omega)=2 \pi R$. The ball $\bar{B}_{R}$ is also the unique minimizer of $\mathcal{I}_{1}$ among the sets $\Omega \in \mathcal{A}$ with $\mathcal{H}^{1}(\partial \Omega)=2 \pi R$.

Turning now to the minimizers of $\mathcal{E}_{\lambda}^{Q}$ over $\mathcal{A}_{m}$, we have the following result:

THEOREM 2.4. Let $\lambda>0, m>0, R=\sqrt{m / \pi}$, and define

$$
\lambda_{0}^{Q}:=\frac{4 m}{\pi} .
$$

Then:

(i) The closed ball $\bar{B}_{R}$ is the unique (up to translation) minimizer of $\mathcal{E}_{\lambda}^{Q}$ over $\mathcal{A}_{m}$ if $\lambda \leq \lambda_{0}^{Q}$.

(ii) There is no minimizer of $\mathcal{E}_{\lambda}^{Q}$ over $\mathcal{A}_{m}$ if $\lambda>\lambda_{0}^{Q}$.

One should contrast the result in Theorem 2.4 with the expectation in the case of nonlocal isoperimetric problems with uniform charge that balls are the unique minimizers, unless it becomes advantageous to split a ball into two equal-size balls [17, 36, 44]. In our problem the latter would happen at the value of $\lambda$ at which two balls of area $\frac{m}{2}$ infinitely far apart and carrying equal charge have the same total energy as one ball of area $m$ and the same total charge. By Lemma 3.5, this is the case if $\lambda>\lambda_{c 1}^{Q}$, where

$$
\lambda_{c 1}^{Q}:=\frac{4 m \sqrt{2}}{\pi}
$$


Comparing this value with $\lambda_{0}^{Q}$, one sees, however, that balls become unstable $b e$ fore it becomes energetically favorable to split them into two equal balls. Furthermore, by Lemma 3.5 the instability with respect to elongations occurs at even higher values of $\lambda>\lambda_{c 2}^{Q}$, where

$$
\lambda_{c 2}^{Q}:=\frac{12 m}{\pi}
$$

An inspection of the proof of Theorem 2.1 shows that the nature of the instability of balls at $\lambda=\lambda_{0}^{Q}$ is, in fact, very different from the two just described: as soon as $\lambda>\lambda_{0}^{Q}$, it becomes energetically favorable to split off many small balls and move them far apart. Those balls will have a small total area but carry a finite fraction of the total charge. This nonlinear instability is closely related to the ill-posedness of the three-dimensional capacitary problem [33,43].

Observe that, as with minimizing $\mathcal{E}_{\lambda}^{Q}$ over $\mathcal{A}$, the infimum over $\mathcal{A}_{m}$ for $\lambda \leq \lambda_{0}^{Q}$ may also be achieved by a sequence of many balls moving off to infinity, as in Remark 2.2. By (2.5), in this case we have

$$
\inf _{\Omega \in \mathcal{A}_{m}} \mathcal{E}_{\lambda}^{Q}(\Omega)=\sqrt{4 \pi m}+\lambda \pi \sqrt{\frac{\pi}{4 m}} \quad \forall \lambda \leq \lambda_{0}^{Q} .
$$

On the other hand, when the infimum is not attained, we can still characterize the optimal scaling of the energy in terms of $\lambda$.

THEOREM 2.5. Let $m>0$ and let $\lambda>\lambda_{0}^{Q}$, where $\lambda_{0}^{Q}$ is defined in (2.7). Then

$$
\inf _{\Omega \in \mathcal{A}_{m}} \mathcal{E}_{\lambda}^{Q}(\Omega)=2 \pi \sqrt{\lambda}
$$

Thus, we have $\inf _{\Omega \in \mathcal{A}_{m}} \mathcal{E}_{\lambda}^{Q}(\Omega) \sim \max \{\sqrt{m}, \sqrt{\lambda}\}$. Note that in physical terms this result means that the minimal energy is linear in charge for all sufficiently large charges. In this case the infimum of the energy is achieved by a union of a ball carrying the fraction

$$
\sqrt{\lambda_{0}^{Q} / \lambda}
$$

of the total charge whose area converges to $m$ and many small balls with vanishing total area and carrying the fraction

$$
\sqrt{\left(\lambda-\lambda_{0}^{Q}\right) / \lambda}
$$

of the total charge, far apart.

We conclude by turning our attention to the problem associated with the energy $\mathcal{E}_{\lambda}^{U}$ from (1.10). One key difference between $\mathcal{E}_{\lambda}^{U}$ and $\mathcal{E}_{\lambda}^{Q}$ is that the former is not bounded from below a priori. Consider the energy of a ball of radius $R$ that equals

$$
\mathcal{E}_{\lambda}^{U}\left(\bar{B}_{R}\right)=2 \pi R-\frac{2 \lambda R}{\pi},
$$


and observe a marked difference between this expression and the one in 2.5, which has a unique minimum as a function of $R$. Here, instead, the energy is a one-homogeneous function of $R$ vanishing at $\lambda=\lambda_{0}^{U}$, where $\lambda_{0}^{U}:=\pi^{2}$. At $\lambda=\lambda_{0}^{U}$ any ball is a minimizer of the energy (among balls), while for $\lambda<\lambda_{0}^{U}$ the infimum of the energy is attained by a sequence of shrinking balls, and for $\lambda>\lambda_{0}^{U}$ the energy is not bounded from below. This picture remains valid also among all sets in $\mathcal{A}$.

THEOREM 2.6. Let $\lambda>0$ and define

$$
\lambda_{0}^{U}:=\pi^{2} .
$$

Then:

(i) If $\lambda<\lambda_{0}^{U}$, we have $\inf _{\Omega \in \mathcal{A}} \mathcal{E}_{\lambda}^{U}(\Omega)=0$, and the infimum is not attained.

(ii) If $\lambda=\lambda_{0}^{U}$, then $\inf _{\Omega \in \mathcal{A}} \mathcal{E}_{\lambda}^{U}(\Omega)=0$, and the infimum is attained by any closed ball and no other set.

(iii) If $\lambda>\lambda_{0}^{U}$, we have $\inf _{\Omega \in \mathcal{A}} \mathcal{E}_{\lambda}^{U}(\Omega)=-\infty$.

Similarly, in the case of minimizing over $\mathcal{A}_{m}$ we have the following result.

THEOREM 2.7. Let $\lambda>0, m>0$, and $R=\sqrt{m / \pi}$, and let $\lambda_{0}^{U}$ be defined in (2.13). Then:

(i) If $\lambda \leq \lambda_{0}^{U}$, then $\bar{B}_{R}$ is the unique minimizer (up to translations) of $\mathcal{E}_{\lambda}^{U}$ over $\mathcal{A}_{m}$.

(ii) If $\lambda>\lambda_{0}^{U}$, then $\inf _{\Omega \in \mathcal{A}} \mathcal{E}_{\lambda}^{U}(\Omega)=-\infty$.

In some sense, the problem associated with $\mathcal{E}_{\lambda}^{U}$ is simpler, because both the perimeter and the capacitary term are one-homogeneous functions with respect to dilations, making the problem very degenerate.

\section{Preliminary Results}

\subsection{Basic Facts about the Capacitary Energy}

We start by collecting several results about existence and uniqueness of equilibrium measures on admissible sets.

Lemma 3.1. Let $\Omega \in \mathcal{A}$. Then there exists a unique probability measure $\mu$ over $\mathbb{R}^{2}$ supported on $\Omega$ such that

$$
\mathcal{I}_{1}(\Omega)=\iint_{\Omega} \frac{d \mu(x) d \mu(y)}{|x-y|} .
$$

Furthermore, $\mu(\partial \Omega)=0$, and we have $d \mu(x)=\rho(x) d x$ for some $\rho \in L^{1}(\Omega)$ satisfying $0<\rho(x) \leq C / \operatorname{dist}(x, \partial \Omega)$ for some constant $C>0$ and all $x \in \operatorname{int}(\Omega)$.

PROOF. For the existence and uniqueness of the equilibrium measure $\mu$, we refer to [40, pp. 131-133]. By [40, theorems 3.13 and 3.14], we have $\mu(\Sigma)=0$ for any set $\Sigma$ such that $\mathcal{H}^{1}(\Sigma)<\infty$, so that in particular $\mu(\partial \Omega)=0$. 
To show that the equilibrium measure $\mu$ is absolutely continuous with respect to the Lebesgue measure in the plane and has a strictly positive density, we recall that the Riesz potential $v: \mathbb{R}^{2} \rightarrow[0, \infty]$ associated with $\Omega$,

$$
v(x):=\int_{\Omega} \frac{d \mu(y)}{|x-y|},
$$

is equal to $\mathcal{I}_{1}(\Omega)$ in $\Omega$ up to a set of zero 1-Riesz capacity and satisfies $v(x) \leq$ $\mathcal{I}_{1}(\Omega)$ for all $x \in \mathbb{R}^{2}$ [40, theorem 2.6 and p. 137]. Therefore, by [40, theorem 3.13] we have $v(x)=\mathcal{I}_{1}(\Omega)$ for a.e. $x \in \Omega$.

Let now $\phi \in C_{c}^{\infty}\left(\mathbb{R}^{2}\right)$ and $\psi \in C^{\infty}\left(\mathbb{R}^{2}\right) \cap L^{1}\left(\mathbb{R}^{2}\right)$ be such that $(-\Delta)^{1 / 2} \phi=$ $\psi$, which is equivalent to [19, lemma 3.2 and prop. 3.3]

$$
\frac{1}{4 \pi} \int_{\mathbb{R}^{2}} \frac{2 \phi(x)-\phi(x-y)-\phi(x+y)}{|y|^{3}} d y=\psi(x) \quad \forall x \in \mathbb{R}^{2} .
$$

Multiplying (3.2) by $\psi$ and integrating over $\mathbb{R}^{2}$, from the Fubini-Tonelli theorem we obtain

$$
\begin{aligned}
& \frac{1}{4 \pi} \iint_{\mathbb{R}^{2}} \frac{v(x)(2 \phi(x)-\phi(x-y)-\phi(x+y))}{|y|^{3}} d x d y= \\
& 2 \pi \int_{\Omega} \phi(x) d \mu(x),
\end{aligned}
$$

where we also took into account that $\phi(x)=\frac{1}{2 \pi} \int_{\mathbb{R}^{2}} \frac{\psi(y)}{|x-y|} d y$ for every $x \in$ $\mathbb{R}^{2}$ [42, lemma 1.3]. Note that (3.4) is the distributional form of the equation $(-\Delta)^{1 / 2} v=2 \pi \mu$.

Finally, observe that for any $\phi$ with support in the interior of $\Omega$ we have

$$
\begin{aligned}
\frac{1}{4 \pi} & \iint_{\mathbb{R}^{2}} \frac{v(x)(2 \phi(x)-\phi(x-y)-\phi(x+y))}{|y|^{3}} d x d y \\
& =\lim _{r \rightarrow 0} \frac{1}{4 \pi} \int_{\mathbb{R}^{2} \backslash B_{r}(0)}\left(\int_{\mathbb{R}^{2}} \frac{v(x)(2 \phi(x)-\phi(x-y)-\phi(x+y))}{|y|^{3}} d x\right) d y \\
& =\lim _{r \rightarrow 0} \frac{1}{4 \pi} \int_{\mathbb{R}^{2} \backslash B_{r}(0)}\left(\int_{\mathbb{R}^{2}} \frac{\phi(x)(2 v(x)-v(x-y)-v(x+y))}{|y|^{3}} d x\right) d y \\
& =\frac{1}{4 \pi} \int_{\Omega} \phi(x)\left(\int_{\mathbb{R}^{2}} \frac{2 v(x)-v(x-y)-v(x+y)}{|y|^{3}} d y\right) d x \\
& =: \int_{\Omega} \phi(x) \rho(x) d x,
\end{aligned}
$$

where we took into account that the inner integrand in the last line of (3.5) is 0 for a.e. $y \in B_{r}(0)$ with $r:=\operatorname{dist}(\operatorname{supp} \phi, \partial \Omega)$. Thus $d \mu(x)=\rho(x) d x$, and the rest of the claims follows from the definition of $\rho$, where we note that the integrand in the last line of (3.5) is strictly positive on a set of positive measure and is bounded above by $2 \mathcal{I}_{1}(\Omega) /|y|^{3}$ for all $y \in \mathbb{R}^{2} \backslash B_{r}(0)$. 
We next state a result about the capacitary energies of unions of disjoint sets from $\mathcal{A}$.

Lemma 3.2. Let $\Omega_{1} \in \mathcal{A}$ and $\Omega_{2} \in \mathcal{A}$ be two disjoint sets, and let $\theta \in[0,1]$. Then

$$
\mathcal{I}_{1}\left(\Omega_{1} \cup \Omega_{2}\right) \leq \theta^{2} \mathcal{I}_{1}\left(\Omega_{1}\right)+(1-\theta)^{2} \mathcal{I}_{2}\left(\Omega_{2}\right)+\frac{2 \theta(1-\theta)}{\operatorname{dist}\left(\Omega_{1}, \Omega_{2}\right)} .
$$

Moreover, there exists $\bar{\theta} \in(0,1)$ such that

$$
\mathcal{I}_{1}\left(\Omega_{1} \cup \Omega_{2}\right)>\bar{\theta}^{2} \mathcal{I}_{1}\left(\Omega_{1}\right)+(1-\bar{\theta})^{2} \mathcal{I}_{2}\left(\Omega_{2}\right) \text {. }
$$

Proof. Let $\theta \in[0,1]$, and let $\mu_{1}$ and $\mu_{2}$ be the equilibrium measures for $\mathcal{I}_{1}\left(\Omega_{1}\right)$ and $\mathcal{I}_{1}\left(\Omega_{2}\right)$, respectively. Then $\tilde{\mu}=\theta \mu_{1}+(1-\theta) \mu_{2}$ is a probability measure supported on $\Omega_{1} \cup \Omega_{2}$. Hence

$$
\begin{aligned}
\mathcal{I}_{1}\left(\Omega_{1} \cup \Omega_{2}\right) \leq & \iint_{\Omega_{1} \cup \Omega_{2}} \frac{d \tilde{\mu}(x) d \tilde{\mu}(y)}{|x-y|} \\
= & \theta^{2} \iint_{\Omega_{1}} \frac{d \mu_{1}(x) d \mu_{1}(y)}{|x-y|}+(1-\theta)^{2} \iint_{\Omega_{2}} \frac{d \mu_{2}(x) d \mu_{2}(y)}{|x-y|} \\
& +2 \theta(1-\theta) \int_{\Omega_{2}} \int_{\Omega_{1}} \frac{d \mu_{1}(x) d \mu_{2}(y)}{|x-y|} \\
\leq & \theta^{2} \mathcal{I}_{1}\left(\Omega_{1}\right)+(1-\theta)^{2} \mathcal{I}_{1}\left(\Omega_{2}\right)+\frac{2 \theta(1-\theta)}{\operatorname{dist}\left(\Omega_{1}, \Omega_{2}\right)}
\end{aligned}
$$

which gives (3.6).

Let now $\mu$ be the equilibrium measure for $\mathcal{I}_{1}\left(\Omega_{1} \cup \Omega_{2}\right)$. Observe that by Lemma 3.1 we have $\mu\left(\Omega_{i}\right)>0$ for $i=1,2$ in view of the fact that the sets $\Omega_{i}$ have nonempty interiors. Define the probability measures $\mu_{i}:=\left.\mu^{-1}\left(\Omega_{i}\right) \mu\right|_{\Omega_{i}}$, and let $\bar{\theta}:=\mu\left(\Omega_{1}\right) \in(0,1)$. Then it holds that

$$
\begin{aligned}
\mathcal{I}_{1}\left(\Omega_{1} \cup \Omega_{2}\right)= & \iint_{\Omega_{1} \cup \Omega_{2}} \frac{d \mu(x) d \mu(y)}{|x-y|} \\
= & \mu^{2}\left(\Omega_{1}\right) \iint_{\Omega_{1}} \frac{d \mu_{1}(x) d \mu_{1}(y)}{|x-y|}+\mu^{2}\left(\Omega_{2}\right) \iint_{\Omega_{2}} \frac{d \mu_{2}(x) d \mu_{2}(y)}{|x-y|} \\
& +2 \mu\left(\Omega_{1}\right) \mu\left(\Omega_{2}\right) \int_{\Omega_{2}} \int_{\Omega_{1}} \frac{d \mu_{1}(x) d \mu_{2}(y)}{|x-y|} \\
> & \bar{\theta}^{2} \mathcal{I}_{1}\left(\Omega_{1}\right)+(1-\bar{\theta})^{2} \mathcal{I}_{1}\left(\Omega_{2}\right),
\end{aligned}
$$

which gives (3.7).

The first result in Lemma 3.2 basically says that the capacitary energy of a union of two admissible sets may be estimated from above by the self-energies of the components with an arbitrary partition of charges plus an interaction energy in terms of the (positive) distance between these sets. Similarly, the second result says that the capacitary energy may be estimated from below by the sum of the capacitary energies for a suitable distribution of charges. 


\subsection{Spherical and Elliptical Domains}

We continue by summarizing some well-known facts about the Riesz capacity of balls and elliptical sets.

LEMMA 3.3. Let

$$
\Omega_{e}=\left\{(x, y): \frac{x^{2}}{a^{2}}+\frac{y^{2}}{b^{2}} \leq 1\right\}
$$

be an ellipse with eccentricity $e=\sqrt{1-(b / a)^{2}}$, where $a$ and $b$ are the major and minor semiaxes, respectively. Then

$$
\mathcal{H}^{1}\left(\partial \Omega_{e}\right)=4 a \mathrm{E}\left(e^{2}\right) \text { and } \mathcal{I}_{1}\left(\Omega_{e}\right)=a^{-1} \mathrm{~K}\left(e^{2}\right),
$$

where $\mathrm{K}(m)$ and $\mathrm{E}(m)$ are the complete elliptic integrals of the first and second kind, respectively [1]. In particular, when $a=b$ we have $\mathcal{I}_{1}\left(\Omega_{e}\right)=\frac{\pi}{2 a}$. The equilibrium measure $\mu$ for $\mathcal{I}_{1}\left(\Omega_{e}\right)$ is given by

$$
d \mu(x, y)=\frac{1}{2 \pi \sqrt{a^{2} b^{2}-b^{2} x^{2}-a^{2} y^{2}}} d x d y .
$$

In addition, we have

$$
\mathcal{I}_{1}\left(\Omega_{e}\right) \leq\left(\frac{\pi^{5}}{4\left|\Omega_{e}\right| \mathcal{H}^{1}\left(\partial \Omega_{e}\right)}\right)^{1 / 3}
$$

PROOF. Most of the formulas in this lemma are well-known. For the reader's convenience, we present some details regarding the Riesz capacitary energy of an elliptical set. The value of $\mathcal{I}_{1}\left(\Omega_{e}\right)$ and the expression for the equilibrium measure $\mu$ follow by passing to a suitable limit from the corresponding expressions for the ellipsoid, which can be found by an explicit solution of the Laplace's equation in three dimensions in ellipsoidal coordinates [39].

The formula in (3.13) is motivated by [20], where it was shown that

$$
\left(\frac{4\left|\Omega_{e}\right| \mathcal{H}^{1}\left(\partial \Omega_{e}\right) \mathcal{I}_{1}^{3}\left(\Omega_{e}\right)}{\pi^{5}}\right)^{1 / 3}=1+O\left(e^{8}\right),
$$

and the inequality can be seen numerically for any particular value of $e \in(0,1)$. To obtain the upper bound on the whole interval is not straightforward, however, since many cancellations give rise to (3.14).

The proof of (3.13) amounts to showing that the function

$$
f(x):=\frac{16 \sqrt{1-x} \mathrm{~K}^{3}(x) \mathrm{E}(x)}{\pi^{4}}
$$

is bounded above by 1 for all $x \in(0,1)$. For this, we differentiate $f$ to obtain, using the identities associated with the complete elliptic integrals [1],

$$
f^{\prime}(x)=\frac{8 \mathrm{~K}^{2}(x)\left(3 \mathrm{E}^{2}(x)-(2-x) \mathrm{E}(x) \mathrm{K}(x)-(1-x) \mathrm{K}^{2}(x)\right)}{\pi^{4} x \sqrt{1-x}} .
$$


Therefore, our proof is concluded once we prove that the function

$$
g(x):=3 \mathrm{E}(x)-(2-x) \mathrm{K}(x)-(1-x) \frac{\mathrm{K}^{2}(x)}{\mathrm{E}(x)}
$$

is negative for all $x \in(0,1)$. This is done by using the estimates

$$
\mathrm{K}(x) \geq \mathrm{K}_{15}(x), \quad \mathrm{E}(x) \leq \mathrm{E}_{15}(x),
$$

where $\mathrm{K}_{15}(x)$ and $\mathrm{E}_{15}(x)$ are the 15th-order Taylor polynomials for $\mathrm{K}(x)$ and $\mathrm{E}(x)$, respectively, obtained by truncating the power series representations of $\mathrm{K}(x)$ and $\mathrm{E}(x)$. Substituting (3.18) into (3.17), we find that $g(x) \leq x^{4} P_{27}(x) / Q_{15}(x)$, where $P_{27}(x)$ and $Q_{15}(x)$ are two explicit polynomials of degrees 27 and 15, respectively, with $P_{27}(0)>0$ and $Q_{15}(0)<0$. The proof is concluded by verifying that both $P_{27}$ and $Q_{15}$ do not change sign in $[0,1]$. All these tedious computations were carried out using computer algebra software Mathematica ${ }^{\circledR} 10.4$.

We now observe that since for a given $\Omega \in \mathcal{A}_{m}$ it is always possible to find $e \in[0,1)$ such that $\Omega_{e} \in \mathcal{A}_{m}$ and $\mathcal{H}^{1}\left(\partial \Omega_{e}\right)=\mathcal{H}^{1}(\partial \Omega)$, by comparing the energy of $\Omega$ with that of $\Omega_{e}$ we obtain the following result from 3.13.

COROLlary 3.4. Let $\lambda>0, m>0$, and $\Omega \in \mathcal{A}_{m}$ be a minimizer of $\mathcal{E}_{\lambda}^{Q}$ over $\mathcal{A}_{m}$. Then

$$
\mathcal{E}_{\lambda}^{Q}(\Omega) \leq \mathcal{H}^{1}(\partial \Omega)+\lambda\left(\frac{\pi^{5}}{4 m \mathcal{H}^{1}(\partial \Omega)}\right)^{1 / 3} .
$$

Note that numerically the right-hand side of (3.13) gives a remarkably accurate value of $\mathcal{I}_{1}\left(\Omega_{e}\right)$ for practically all values of $e[20]$. For example, when $e=0.7$, it predicts the value of $\mathcal{I}_{1}\left(\Omega_{e}\right)$ with a relative error of $5 \times 10^{-5}$. Even at $e=$ 0.9999 the relative error of the leading-order expression is within about $25 \%$. Let us also point out that the leading-order term in 3.13 was found to be a very good approximation for the values of $\mathcal{I}_{1}$ for sets of varied simple shapes, not limited to elliptical sets [20]. In fact, if 3.19] were an equality, one would immediately conclude from the isoperimetric inequality that balls are the only minimizers of $\mathcal{E}_{\lambda}^{Q}$ over $\mathcal{A}_{m}$ for all $\lambda \leq \lambda_{c 2}^{Q}$, where $\lambda_{c 2}^{Q}$ is given by (2.9).

Recalling Lemma 3.2, the computations of Lemma 3.3 allow us to make conclusions about instabilities of balls.

LemmA 3.5. Let $m>0, R=\sqrt{m / \pi}$, and $\lambda_{c 1}^{Q}$ and $\lambda_{c 2}^{Q}$ be as in 2.8) and 2.9. Then

Please insert missing right parenthesis in (i). (i) If $\lambda>\lambda \underset{c 1}{Q}$, a ball with area $m$ is unstable with respect to splitting, i.e., $\mathcal{E}_{\lambda}^{Q}\left(\bar{B}_{R}\right)>\mathcal{E}_{\lambda}^{Q}\left(\bar{B}_{R / \sqrt{2}}(0) \cup \bar{B}_{R / \sqrt{2}}\left(x_{0}\right)\right.$ for all $x_{0} \in \mathbb{R}^{2}$ with $\left|x_{0}\right| \gg R$.

(ii) If $\lambda>\lambda_{c 1}^{Q}$, a ball with area $m$ is unstable with respect to elongations, i.e., there exists an elliptical set $\Omega_{e}$ such that $\mathcal{E}_{\lambda}^{Q}\left(\bar{B}_{R}\right)>\mathcal{E}_{\lambda}^{Q}\left(\Omega_{e}\right)$. 
PROOF. The first assertion follows by a straightforward computation, using Lemma 3.3 and Lemma 3.2. For the second, we use Corollary 3.4, minimizing the right-hand side of (3.19) with respect to $\mathcal{H}^{1}(\partial \Omega) \geq 2 \pi R$.

We now show that a single ball has lower energy than a disjoint union of balls with the same total perimeter.

LEMMA 3.6. Let $\Omega=\bigcup_{i=1}^{N} \bar{B}_{r_{i}}\left(x_{i}\right)$ be a union of a finite number of disjoint closed balls, and let $r:=\sum_{i=1}^{N} r_{i}$. Then

$$
\mathcal{E}_{\lambda}^{Q}\left(\bar{B}_{r}\right) \leq \mathcal{E}_{\lambda}^{Q}(\Omega)
$$

with equality if and only if $\Omega=\bar{B}_{r}(x)$ for some $x \in \mathbb{R}^{2}$.

PROOF. If $\Omega$ is a ball then there is nothing to prove.

Assume that $\Omega=\bar{B}_{r_{1}}\left(x_{1}\right) \cup \bar{B}_{r_{2}}\left(x_{2}\right)$ is the union of two disjoint balls. We show that the ball $\bar{B}_{r}$, with $r_{1}+r_{2}=r$, always has less energy. Indeed, for every $\theta \in[0,1]$ it holds that

$$
\frac{1}{r_{1}+r_{2}} \leq \frac{\theta^{2}}{r_{1}}+\frac{(1-\theta)^{2}}{r_{2}}
$$

which is readily verified by noticing that $\hat{\theta}=r_{1} /\left(r_{1}+r_{2}\right)$ minimizes the right-hand side of (3.21), and equality holds for $\theta=\hat{\theta}$. Recalling that $\mathcal{I}_{1}\left(\bar{B}_{r}\right)=\pi /(2 r)$, inequality (3.21) can be rephrased as

$$
\mathcal{I}_{1}\left(\bar{B}_{r}\right) \leq \theta^{2} \mathcal{I}_{1}\left(\bar{B}_{r_{1}}\left(x_{1}\right)\right)+(1-\theta)^{2} \mathcal{I}_{1}\left(\bar{B}_{r_{2}}\left(x_{2}\right)\right) \quad \forall \theta \in[0,1] .
$$

On the other hand, since $\mathcal{H}^{1}\left(\partial B_{r}\right)=\mathcal{H}^{1}\left(\partial B_{r_{1}}\left(x_{1}\right)\right)+\mathcal{H}^{1}\left(\partial B_{r_{2}}\left(x_{2}\right)\right)$ from 3.22) and (3.7), it follows that

$$
\begin{aligned}
\mathcal{E}_{\lambda}^{Q}\left(\bar{B}_{r}\right) & \leq \mathcal{E}_{\lambda \bar{\theta}^{2}}^{Q}\left(\bar{B}_{r_{1}}\left(x_{1}\right)\right)+\mathcal{E}_{\lambda(1-\bar{\theta})^{2}}^{Q}\left(\bar{B}_{r_{2}}\left(x_{2}\right)\right) \\
& <\mathcal{E}_{\lambda}^{Q}\left(\bar{B}_{r_{1}}\left(x_{1}\right) \cup \bar{B}_{r_{2}}\left(x_{2}\right)\right) .
\end{aligned}
$$

Assume now that $\Omega$ consists of more than two disjoint balls. In this case we write $\Omega=\Omega^{\prime} \cup \Omega^{\prime \prime}$, where $\Omega^{\prime}:=\bar{B}_{r_{1}}\left(x_{1}\right) \cup \bar{B}_{r_{2}}\left(x_{2}\right)$ and $\Omega^{\prime \prime}:=\Omega \backslash \Omega^{\prime} \neq \varnothing$. Again, from Lemma 3.2 we know that there exists $\bar{\theta} \in(0,1)$ and $\delta>0$ such that

$$
\mathcal{I}_{1}(\Omega) \geq \bar{\theta}^{2} \mathcal{I}_{1}\left(\Omega^{\prime}\right)+(1-\bar{\theta})^{2} \mathcal{I}_{1}\left(\Omega^{\prime \prime}\right)+\delta .
$$

Moreover, from the previous discussion it follows that $\mathcal{I}_{1}\left(\Omega^{\prime}\right) \geq \mathcal{I}_{1}\left(\bar{B}_{\bar{r}}(x)\right)$, where $\bar{r}:=r_{1}+r_{2}$ for any $x \in \mathbb{R}^{2}$, so that we get

$$
\mathcal{I}_{1}(\Omega) \geq \bar{\theta}^{2} \mathcal{I}_{1}\left(\bar{B}_{\bar{r}}(x)\right)+(1-\bar{\theta})^{2} \mathcal{I}_{1}\left(\Omega^{\prime \prime}\right)+\delta .
$$

Recalling (3.6), we then obtain the estimate

$$
\begin{aligned}
\mathcal{I}_{1}(\Omega) & \geq \bar{\theta}^{2} \mathcal{I}_{1}\left(\bar{B}_{\bar{r}}(x)\right)+(1-\bar{\theta})^{2} \mathcal{I}_{1}\left(\Omega^{\prime \prime}\right)+\delta \\
& \geq \mathcal{I}_{1}\left(\bar{B}_{\bar{r}}(x) \cup \Omega^{\prime \prime}\right)+\delta-\frac{2 \bar{\theta}(1-\bar{\theta})}{\operatorname{dist}\left(\bar{B}_{\bar{r}}(x), \Omega^{\prime \prime}\right)} .
\end{aligned}
$$


If we choose $x \in \mathbb{R}^{2}$ such that $\operatorname{dist}\left(\bar{B}_{\bar{r}}(x), \Omega^{\prime \prime}\right)>(2 \delta)^{-1}$, we then get $\mathcal{I}_{1}(\Omega)>$ $\mathcal{I}_{1}\left(\bar{B}_{\bar{r}}(x) \cup \Omega^{\prime \prime}\right)$. Recalling that $\mathcal{H}^{1}(\partial \Omega)=\mathcal{H}^{1}\left(\partial B_{\bar{r}}(x) \cup \partial \Omega^{\prime \prime}\right)$, this implies that

$$
\mathcal{E}_{\lambda}^{Q}(\Omega)>\mathcal{E}_{\lambda}^{Q}\left(\bar{B}_{\bar{r}}(x) \cup \Omega^{\prime \prime}\right)
$$

The proof is then easily concluded by means of an inductive argument.

Our next lemma is a version of a well-known Brunn-Minkowski inequality for the Newtonian capacity of convex bodies in $\mathbb{R}^{3}[10,14,18]$.

LEMMA 3.7. For any pair of convex bodies $\Omega_{1}, \Omega_{2}$ in $\mathbb{R}^{2}$ and for any $\lambda \in[0,1]$, it holds that

$$
C_{1}\left(\lambda \Omega_{1}+(1-\lambda) \Omega_{2}\right) \geq \lambda C_{1}\left(\Omega_{1}\right)+(1-\lambda) C_{1}\left(\Omega_{2}\right),
$$

where $C_{1}(\Omega):=\mathcal{I}_{1}^{-1}(\Omega)$ is the 1-Riesz capacity of $\Omega$. Moreover, the equality holds if and only if $\Omega_{1}$ is homothetic to $\Omega_{2}$.

PROOF. The inequality has been proved in [45, theorem 1.1(ii)] and can also be seen as the limit case of the corresponding inequality for the Newtonian capacity of thin cylindrical domains in $\mathbb{R}^{3}[10]$.

To get the characterization of the equality case, we introduce $u: \mathbb{R}^{3} \rightarrow \mathbb{R}$, which is the harmonic extension of $v$ in $(3.2)$ to $\mathbb{R}^{3} \backslash(\Omega \times\{0\})$. Notice that up to a constant factor the function $u$ is the capacitary potential of a convex set $\Omega \times\{0\} \subset$ $\mathbb{R}^{3}$, and the level sets $\left\{x \in \mathbb{R}^{3}: u(x)>t\right\}$ are regular convex bodies in $\mathbb{R}^{3}$ for all $t \in(0,1)$ (see [45, theorem 1.1 (i)]). With this observation, the final part of the proof of [18, theorem 1, pp. 476-478] applies without modifications.

The following is the cornerstone result for the proof of Theorem 2.1. Its proof, in a different setting, was developed in [12].

LEMMA 3.8. Let $\Omega \in \mathcal{A}$ be a convex set. Then $\mathcal{E}_{\lambda}^{Q}(\Omega) \geq \mathcal{E}_{\lambda}^{Q}\left(\bar{B}_{\Omega}\right)$, where $\bar{B}_{\Omega}$ is a ball with the same perimeter as $\Omega$, with equality if and only if $\Omega$ is a closed ball.

[50, theorem 3.3.2] appears to be a chapter in an anthology. Please give citation to chapter by Hadwiger.
PROOF. By a result of Hadwiger [50, theorem 3.3.2], we know that there exists a sequence of Minkowski rotation means of $\Omega$ that converges to a ball in the Hausdorff metric. Namely, there exists a sequence of rotations $\left\{T_{n}\right\}_{n \in \mathbb{N}}$ such that the sets

$$
\Omega_{n}:=\frac{\sum_{k=1}^{n} T_{k}(\Omega)}{n}
$$

converge in the Hausdorff metric to a ball $\bar{B}_{\Omega}$. We note that by Lemma 3.7 we have

$$
\mathcal{I}_{1}\left(\Omega_{n}\right)=\frac{1}{C_{1}\left(\Omega_{n}\right)} \leq \frac{1}{C_{1}(\Omega)}=\mathcal{I}_{1}(\Omega)
$$

Suppose that the equality holds in (3.30) for any $n \in \mathbb{N}$ so that $\Omega_{n}$ is homothetic to $\Omega$. Since $\Omega_{n} \rightarrow \bar{B}_{\Omega}$ in the Hausdorff metric, we get that $\Omega=\bar{B}_{\Omega}$. 
Since the perimeter is linear with respect to the Minkowski sum of convex sets in $\mathbb{R}^{2}$, we have $\mathcal{H}^{1}\left(\partial \bar{B}_{\Omega}\right)=\mathcal{H}^{1}(\partial \Omega)$. Gathering the information above, we get

$$
\begin{aligned}
\mathcal{H}^{1}\left(\partial \bar{B}_{\Omega}\right)+\lambda \mathcal{I}_{1}\left(\bar{B}_{\Omega}\right) & =\lim _{n \rightarrow \infty}\left(\mathcal{H}^{1}\left(\partial \Omega_{n}\right)+\lambda \mathcal{I}_{1}\left(\Omega_{n}\right)\right) \\
& \leq \mathcal{H}^{1}(\partial \Omega)+\lambda \mathcal{I}_{1}(\Omega),
\end{aligned}
$$

with equality if and only if $\Omega=\bar{B}_{\Omega}$. This concludes the proof.

\subsection{Indecomposable Components}

Given a set $\Omega \in \mathcal{A}$, let $\bar{\Omega}^{M}$ be its measure-theoretic closure, namely,

$$
\bar{\Omega}^{M}:=\left\{x \in \mathbb{R}^{2}: \liminf _{r \rightarrow 0} \frac{\left|\Omega \cap B_{r}(x)\right|}{\pi r^{2}}>0\right\} .
$$

Since $P\left(\bar{\Omega}^{M}\right)=P(\Omega)=\mathcal{H}^{1}\left(\partial^{M} \Omega\right) \leq \mathcal{H}^{1}(\partial \Omega)<+\infty$, the set $\bar{\Omega} R^{M}$ is a set of finite perimeter [4]. Therefore, following [3], for a set $\Omega \in \mathcal{A}$ we can write $\bar{\Omega}^{M}=\left(\bigcup_{i} \Omega_{i}\right) \cup \Sigma$, with $\mathcal{H}^{1}(\Sigma)=0$, where the sets $\Omega_{i} \in \mathcal{A}$ are the socalled indecomposable components of $\bar{\Omega}^{M}$ (finitely many or a countable family). In particular, the sets $\Omega_{i}$ admit unique representatives that are connected and satisfy the following properties:

- $\mathcal{H}^{1}\left(\Omega_{i} \cap \Omega_{j}\right)=0$ for $i \neq j$,

- $\left|\bar{\Omega}^{M}\right|=\sum_{i}\left|\Omega_{i}\right|$,

- $P(\Omega)=P\left(\bar{\Omega}^{M}\right)=\sum_{i} P\left(\Omega_{i}\right)$,

- $\Omega_{i}=\overline{\operatorname{int}\left(\Omega_{i}\right)}$.

Moreover, each set $\Omega_{i}$ is indecomposable in the sense that it cannot be further decomposed as above. We refer to these representatives of $\Omega_{i}$ as the connected components of $\Omega \in \mathcal{A}$. Note that such a definition is necessary, since we are dealing with equivalence classes of subsets of $\mathbb{R}^{2}$ for which the usual notion of connectedness is not well-defined (consider, for instance, the union of two touching closed balls, which is equivalent to the same set minus the contact point). We point out, however, that this notion coincides with the standard notion of connected components in the following sense: if $\Omega$ has a regular boundary, for instance, is Lipschitz-continuous, then the components $\Omega_{i}$ are (the closure of) the usual connected components of the interior of $\Omega$.

Observe that $\mathcal{H}^{1}\left(\partial \operatorname{co}\left(\Omega_{i}\right)\right) \leq \mathcal{H}^{1}\left(\partial \Omega_{i}\right)$ for every $i$, where $\operatorname{co}\left(\Omega_{i}\right)$ denotes the convex envelope of $\Omega_{i}$. This follows from the fact that the outer boundary of a connected component can be parametrized by a Jordan curve of finite length (see [3, sec. 8]). In addition, since $\partial \Omega$ is negligible with respect to the equilibrium measure for $\mathcal{I}_{1}(\Omega)$ by Lemma 3.1 , we have $\mathcal{I}_{1}(\Omega)=\mathcal{I}_{1}\left(\bar{\Omega}^{M}\right)$. Lastly, we have $|\Omega|=\left|\bar{\Omega}^{M}\right|$ and $\operatorname{int}(\Omega)=\operatorname{int}\left(\bar{\Omega}^{M}\right)$.

We conclude this section by showing that any admissible set may be replaced by an admissible set with lower energy that consists of only a finite number of connected components. In particular, this result shows that minimizers of $\mathcal{E}_{\lambda}^{Q}$ over $\mathcal{A}$ are connected whenever they exist. We note that replacing an admissible set by 
a set with finitely many connected components as in the lemma below imparts a certain degree of regularity. Namely, our procedure removes the parts of the topological boundary of an admissible set that have positive $\mathcal{H}^{1}$ measure but do not belong to the measure-theoretic closure (e.g., a line segment attached to a ball).

Lemma 3.9. Let $\Omega \in \mathcal{A}$. Then there exists a set $\Omega^{\prime} \in \mathcal{A}$ such that $\Omega^{\prime}$ has a finite number of connected components and

$$
\mathcal{E}_{\lambda}^{Q}\left(\Omega^{\prime}\right) \leq \mathcal{E}_{\lambda}^{Q}(\Omega)
$$

Moreover, the inequality in (3.33) is strict whenever $\Omega$ has more than one connected component.

PROOF. Without loss of generality, we may assume that $\bar{\Omega}^{M}$ has at least two indecomposable components. Let $\bar{\Omega}^{M}=\left(\bigcup_{i \in I} \Omega_{i}\right) \cup \Sigma$ be the decomposition of $\bar{\Omega}^{M}$ into indecomposable components. Let $0<\varepsilon<\frac{1}{2}$ to be fixed later, and let $N \geq 2$, depending on $\varepsilon$, be such that $1-\delta:=\sum_{i=1}^{N} \mu\left(\Omega_{i}\right) \geq 1-\varepsilon$, where $\mu$ is the equilibrium measure of $\Omega$. If $I$ is finite, we let $N$ be the number of indecomposable components of $\Omega$.

For $i \in I$, we let $\xi_{i}$ be the barycenter of $\Omega_{i}$ and set

$$
\Omega^{N}(t):=\bigcup_{i=1}^{N}\left(\Omega_{i}-\xi_{i}+i t e_{1}\right),
$$

where $e_{1}$ denotes the unit vector in the first coordinate direction. Let also $f_{i}^{t}(x):=$ $x-\xi_{i}+i t e_{1}$, and let $\mu_{i}^{t}:=\left.\left(f_{i}^{t}\right)_{\sharp} \mu\right|_{\Omega_{i}}$ be the pushforward of $\mu_{i}:=\left.\mu\right|_{\Omega_{i}}$ through the map $f_{i}^{t}$. By a straightforward computation, we get

$$
\begin{aligned}
\mathcal{I}_{1}(\Omega)-\mathcal{I}_{1}\left(\Omega^{N}(t)\right) \geq & \left(1-\frac{1}{(1-\varepsilon)^{2}}\right) \sum_{i=1}^{N} \iint \frac{d \mu_{i}(x) d \mu_{i}(y)}{|x-y|} \\
& +2 \sum_{i=1}^{N-1} \sum_{j=i+1}^{N} \iint \frac{d \mu_{i}(x) d \mu_{j}(y)}{|x-y|} \\
& -\frac{2}{(1-\varepsilon)^{2}} \sum_{i=1}^{N-1} \sum_{j=i+1}^{N} \iint \frac{d \mu_{i}^{t}(x) d \mu_{j}^{t}(y)}{|x-y|} .
\end{aligned}
$$

Since $\Omega \in \mathcal{A}$, there exists $R>0$ such that $\operatorname{diam}(\Omega)<R$. Moreover, if $t>2 R$ we have that whenever $i \neq j$, for any $(x, y) \in\left(-\xi_{i}+\Omega_{i}+i t e_{1}\right) \times\left(-\xi_{j}+\Omega_{j}+j t e_{1}\right)$, it holds that $|x-y| \geq t-2 R$. Let also

$$
M(\Omega):=2 \sum_{i=1}^{N-1} \sum_{j=i+1}^{N} \iint d \mu_{i}(x) d \mu_{j}(y)>0 .
$$


The last inequality follows from Lemma 3.1. With these choices, we get

$$
\begin{aligned}
\mathcal{I}_{1}(\Omega)-\mathcal{I}_{1}\left(\Omega^{N}(t)\right) & \geq-8 \varepsilon \sum_{i=1}^{N} \iint \frac{d \mu_{i}(x) d \mu_{i}(y)}{|x-y|}+\left(\frac{1}{R}-\frac{4}{t-2 R}\right) M(\Omega) \\
& \geq-8 \varepsilon \mathcal{I}_{1}(\Omega)+\left(\frac{1}{R}-\frac{4}{t-2 R}\right) M(\Omega) \geq \frac{M(\Omega)}{4 R},
\end{aligned}
$$

\section{Proofs of the Main Results}

We turn now to the proofs of Theorems $2.1-2.7$.

Proof of Theorem 2.1. Let $\Omega \in \mathcal{A}$, hence $\mathcal{E}_{\lambda}^{Q}(\Omega)<+\infty$. We first show that there exists a closed ball $\bar{B}$ such that

$$
\mathcal{E}_{\lambda}^{Q}(\Omega) \geq \mathcal{E}_{\lambda}^{Q}(\bar{B})
$$

and equality holds if and only if $\Omega$ itself is a ball.

Thanks to Lemma 3.9 we can suppose that the number of the connected components $\Omega_{i}$ of $\Omega$ is finite. If $\Omega$ has only one connected component, then we can convexify it and use Lemma 3.8. So we suppose that $\Omega$ has at least two connected components.

Let $\operatorname{co}\left(\Omega_{i}\right)$ be the convex envelope of $\Omega_{i}$, and denote by $\mu$ the equilibrium measure of $\Omega$ and by $\mu^{i}$ its restriction to $\Omega_{i}$. Note that $\mu^{i}\left(\Omega_{i}\right)>0$ for all $i$ by Lemma 3.1. Then by Lemma 3.8 we get

$$
\begin{aligned}
\mathcal{E}_{\lambda}^{Q}(\Omega)= & \sum_{i} \mathcal{H}^{1}\left(\partial \Omega_{i}\right)+\lambda \sum_{i} \iint \frac{d \mu^{i}(x) d \mu^{i}(y)}{|x-y|} \\
& +\lambda \sum_{i \neq j} \iint \frac{d \mu^{i}(x) d \mu^{j}(y)}{|x-y|} \\
\geq & \sum_{i} \mathcal{H}^{1}\left(\partial \operatorname{co}\left(\Omega_{i}\right)\right)+\lambda \sum_{i}\left(\mu^{i}\left(\Omega_{i}\right)\right)^{2} \mathcal{I}_{1}\left(\Omega_{i}\right)+\frac{\lambda M(\Omega)}{\operatorname{diam}(\Omega)} \\
\geq & \sum_{i} \mathcal{H}^{1}\left(\partial \operatorname{co}\left(\Omega_{i}\right)\right)+\lambda \sum_{i}\left(\mu^{i}\left(\Omega_{i}\right)\right)^{2} \mathcal{I}_{1}\left(\operatorname{co}\left(\Omega_{i}\right)\right)+\frac{\lambda M(\Omega)}{\operatorname{diam}(\Omega)} \\
\geq & \left.\sum_{i} \mathcal{H}^{1}\left(\partial B^{i}\right)\right)+\lambda \sum_{i}\left(\mu^{i}\left(\Omega_{i}\right)\right)^{2} \mathcal{I}_{1}\left(\overline{B^{i}}\right)+\frac{\lambda M(\Omega)}{\operatorname{diam}(\Omega)} .
\end{aligned}
$$

where $M(\Omega):=\sum_{i \neq j} \mu\left(\Omega_{i}\right) \mu\left(\Omega_{j}\right)>0$ and $B^{i}$ are balls with mutual distance greater than a number $n$ to be fixed later and such that $\mathcal{H}^{1}\left(\partial B^{i}\right)=\mathcal{H}^{1}\left(\partial \Omega_{i}\right)$. In 
the first inequality we used the fact that $\left(\mu^{i}\left(\Omega_{i}\right)\right)^{-1} \mu^{i}$ is admissible in the definition of $\mathcal{I}_{1}\left(\Omega_{i}\right)$, while for the second one we exploited the inequality $\mathcal{I}_{1}\left(\Omega_{i}\right) \geq$ $\mathcal{I}_{1}\left(\operatorname{co}\left(\Omega_{i}\right)\right)$. Let now $\tilde{\mu}^{i}$ be the optimal measure for $B^{i}$. The measure $\sum_{i} \mu\left(\Omega_{i}\right) \tilde{\mu}^{i}$ is then a probability measure on $\bigcup_{i} B^{i}$. Thus

$$
\begin{aligned}
\mathcal{I}_{1}\left(\bigcup_{i} B^{i}\right) \leq & \sum_{i} \mu^{2}\left(\Omega_{i}\right) \iint \frac{d \tilde{\mu}^{i}(x) d \tilde{\mu}^{i}(y)}{|x-y|} \\
& +\sum_{i \neq j} \mu\left(\Omega_{i}\right) \mu\left(\Omega_{j}\right) \iint \frac{d \tilde{\mu}^{i}(x) d \tilde{\mu}^{j}(y)}{|x-y|} \\
\leq & \sum_{i} \mu^{2}\left(\Omega_{i}\right) \mathcal{I}_{1}\left(B^{i}\right)+\frac{\sum_{i \neq j} \mu\left(\Omega_{i}\right) \mu\left(\Omega_{j}\right)}{n} .
\end{aligned}
$$

By (4.2) and 4.3) we get

$$
\mathcal{E}_{\lambda}^{Q}(\Omega) \geq \mathcal{E}_{\lambda}^{Q}\left(\bigcup_{i} B^{i}\right)+\frac{\lambda M(\Omega)}{\operatorname{diam}(-)}-\frac{\lambda M(\Omega)}{n} \geq \mathcal{E}_{\lambda}^{Q}\left(\bigcup_{i} B^{i}\right)
$$

where the last inequality holds if we choose $n$ big enough, depending on $\Omega$.

To conclude, we only have to show that

$$
\mathcal{E}_{\lambda}^{Q}\left(\bigcup_{i} \overline{B^{i}}\right) \geq \mathcal{E}_{\lambda}^{Q}(\bar{B})
$$

for a ball $B$ such that $\mathcal{H}^{1}(\partial B)=\sum_{i} \mathcal{H}^{1}\left(\partial B^{i}\right)$, where equality holds only if the union of balls in the left-hand side of (4.5) is indeed a unique ball. But this is exactly the statement of Lemma 3.6. Once we know that the minimizer of $\mathcal{E}_{\lambda}^{Q}$ in $\mathcal{A}$ is a ball, we conclude by optimizing on the radius of all possible balls.

Proof of Corollary 2.3. The proof is contained in that of Theorem 2.1. where we also note that at every step of the proof the perimeters of the considered sets cannot increase. Therefore, if the perimeter of the final ball $B$ is strictly less than $\mathcal{H}^{1}(\partial \Omega)$, we can further decrease $\mathcal{I}_{1}$ by dilating that ball to ensure that its perimeter equals $\mathcal{H}^{1}(\partial \Omega)$.

Proof of Theorem 2.4. We first prove (i). Notice that if $\lambda=\lambda_{0}^{Q}$, by Theorem 2.1 we know that the ball $\bar{B}_{R}$ is a global minimizer of $\mathcal{E}_{\lambda}^{Q}$ in $\mathcal{A}$. In particular, $\mathcal{E}_{\lambda_{0}^{Q}}^{Q}(\Omega) \geq \mathcal{E}_{\lambda_{0}^{Q}}^{Q}\left(\bar{B}_{R}\right)$, with equality if and only if $\Omega$ is a closed ball. 
Now, since $\mathcal{I}_{1}$ is maximized by balls under volume constraint (see [46, p. 14] and [47, sec. VII.7.3, p. 157], and since $\lambda_{0}^{Q}-\lambda>0$, we get

$$
\begin{aligned}
\mathcal{E}_{\lambda}^{Q}(\Omega) & =\mathcal{E}_{\lambda_{0}^{Q}}^{Q}(\Omega)-\left(\lambda_{0}^{Q}-\lambda\right) \mathcal{I}_{1}(\Omega) \\
& \geq \mathcal{E}_{\lambda_{0}^{Q}}^{Q}\left(\bar{B}_{R}\right)-\left(\lambda_{0}^{Q}-\lambda\right) \mathcal{I}_{1}(\Omega) \\
& \geq \mathcal{E}_{\lambda_{0}^{Q}}^{Q}\left(\bar{B}_{R}\right)-\left(\lambda_{0}^{Q}-\lambda\right) \mathcal{I}_{1}\left(\bar{B}_{R}\right)=\mathcal{E}_{\lambda}^{Q}\left(\bar{B}_{R}\right),
\end{aligned}
$$

with equality if and only if $\Omega$ is a ball; thus $\bar{B}_{R}$ is a minimizer. Moreover, all other minimizers are translates of $\bar{B}_{R}$. Indeed, every minimizer has $B_{R}$ as its interior (up to a translation), and the boundary of the minimizer differs from $\partial B_{R}$ by a set of zero $\mathcal{H}^{1}$ measure.

We now prove (ii). We have to show that there is no minimizer of $\mathcal{E}_{\lambda}^{Q}$ over $\mathcal{A}_{m}$ for $\lambda>\lambda_{0}^{Q}$. We argue by contradiction and assume that there is a minimizer $\Omega \in \mathcal{A}_{m}$ of $\mathcal{E}_{\lambda}^{Q}$. In view of the statement of Theorem 2.1, we have $\mathcal{E}_{\lambda}^{Q}(\Omega)>$ $2 \pi \sqrt{\lambda}$. We now consider a competitor set $\widetilde{\Omega}=\bar{B}_{\widetilde{R}}(0) \cup\left(\bigcup_{i=1}^{N} \bar{B}_{r / N}\left(x_{i}\right)\right)$, where $N \in \mathbb{N}, r>0$ is to be determined, $\widetilde{R}=\sqrt{m / \pi-r^{2} / N}$, and $x_{i} \in \mathbb{R}^{2}$ with $\left|x_{i}-x_{j}\right| \gg \widetilde{R}$ for any $i \neq j$. As in Lemma 3.2 the energy of $\widetilde{\Omega}$ may be estimated from above by partitioning the total charge between $\bar{B}_{\widetilde{R}}(0)$ and $\bar{B}_{r / N}\left(x_{i}\right)$. Suppose that the fraction of the total charge on each ball $\bar{B}_{r / N}\left(x_{i}\right)$ is equal to $\theta / N$ for some $\theta \in(0,1)$. We choose $r=\frac{1}{2} \theta \sqrt{\lambda}$ and $\theta$ in such a way that the charge fraction $1-\theta$ on $\bar{B}_{\widetilde{R}}(0)$ is optimal for a ball of radius $\widetilde{R}$ according to Theorem 2.1. Then we get

$$
\lambda(1-\theta)^{2}=4 \widetilde{R}^{2}=\lambda_{0}^{Q}-\frac{\theta^{2} \lambda}{N},
$$

which for $\lambda>\lambda_{0}^{Q}$ has a unique solution for $\theta$ whenever $N$ is sufficiently large. On the other hand, by Lemma 3.2 with our choices of the charge partitioning we have, for any $\varepsilon>0$ and $x_{i}$ sufficiently far apart,

$$
\begin{aligned}
\mathcal{E}_{\lambda}^{Q}(\widetilde{\Omega}) & \leq \mathcal{E}_{\lambda(1-\theta)^{2}}^{Q}\left(\bar{B}_{R_{0}}(0)\right)+\sum_{i=1}^{N} \mathcal{E}_{\lambda \theta^{2} / N^{2}}^{Q}\left(\bar{B}_{r / N}\left(x_{i}\right)\right)+\varepsilon \\
& =2 \pi(1-\theta) \sqrt{\lambda}+\sum_{i=1}^{N} \frac{2 \pi \theta \sqrt{\lambda}}{N}+\varepsilon=2 \pi \sqrt{\lambda}+\varepsilon .
\end{aligned}
$$

In view of the arbitrariness of $\varepsilon$, this contradicts the minimality of $\mathcal{E}_{\lambda}^{Q}(\Omega)>$ $2 \pi \sqrt{\lambda}$.

Proof OF THEOREM 2.5. Just use as a competitor the set $\widetilde{\Omega}$ constructed in the proof of Theorem 2.4 point (ii), to show that for any $\varepsilon>0$ there exists a set whose 
energy is lower than $2 \pi \sqrt{\lambda}+\varepsilon$. By Theorem 2.1 its energy is greater than $2 \pi \sqrt{\lambda}$; thus we conclude.

Proof of Theorem 2.6. By Corollary 2.3 we know that for all $\Omega \in \mathcal{A}$ we have $\mathcal{E}_{\lambda}^{U}(\Omega) \geq \mathcal{E}_{\lambda}^{U}\left(\bar{B}_{\Omega}\right)$, where $\bar{B}_{\Omega}$ is a ball with the same perimeter as $\Omega$, with equality if and only if $\Omega$ is a ball. As the energy of a ball of radius $R$ is given in (2.12), this immediately implies that if $\lambda=\lambda_{0}^{U}$, then the energy of any ball is 0 . In particular, statement (ii) follows.

If $\lambda>\lambda_{0}^{U}$, by (2.12) the energy of a ball of radius $R$ diverges to $-\infty$ as $R \rightarrow$ $+\infty$, proving statement (iii). To prove (i), let $\lambda<\lambda_{0}^{U}$ and recall that $\mathcal{I}_{1}^{-1}$ is minimized by balls under volume constraint. Then we have that, for any $\Omega \in \mathcal{A}$, it holds that

$$
\begin{aligned}
\mathcal{E}_{\lambda}^{U}(\Omega) & =\mathcal{E}_{\lambda_{0}^{U}}^{U}(\Omega)+\left(\lambda_{0}^{U}-\lambda\right) \mathcal{I}_{1}^{-1}(\Omega) \\
& \geq \mathcal{E}_{\lambda_{0}^{U}}^{U}\left(\bar{B}_{R}\right)+\left(\lambda_{0}^{U}-\lambda\right) \mathcal{I}_{1}^{-1}\left(\bar{B}_{R}\right) \\
& =\mathcal{E}_{\lambda}^{U}\left(\bar{B}_{R}\right)=\left(\lambda_{0}^{U}-\lambda\right) \frac{2 R}{\pi}>0,
\end{aligned}
$$

where $\bar{B}_{R}$ is a ball with the same volume as $\Omega$. Therefore, the infimum of $\mathcal{E}_{\lambda}^{U}$ is 0 , and it is not attained.

Proof of TheOREm 2.7, By (4.9) and Theorem 2.6(ii) we immediately obtain (i). Let us prove (ii). For $n \in \mathbb{N}$ and $d>0$ sufficiently large, we let $r_{n}:=\sqrt{m /(\pi n)}$ and

$$
\Omega_{n, d}:=\bigcup_{i=1}^{n} B_{r_{n}}\left(x_{i}\right),
$$

where the centers $x_{i} \in \mathbb{R}^{2}$ are chosen in such a way that $\left|x_{i}-x_{j}\right| \geq d$ for $i \neq j$. Thus $\left|\Omega_{n, d}\right|=m$, and by Lemma 3.2 its asymptotic energy as $d \rightarrow \infty$ can be estimated as

$$
\lim \sup _{d \rightarrow \infty} \mathcal{E}_{\lambda}^{U}\left(\Omega_{n, d}\right) \leq n \mathcal{E}_{\lambda}^{U}\left(\bar{B}_{r_{n}}\right)=2 \sqrt{\pi m}\left(1-\frac{\lambda}{\pi^{2}}\right) \sqrt{n}
$$

Since $\lambda>\pi^{2}$, the latter diverges to $-\infty$ as $n \rightarrow \infty$.

Acknowledgments. The authors wish to thank M. Goldman, J. Lamboley, and V. Moroz for helpful discussions on the subject. The work of CBM was supported, in part, by National Science Foundation grants DMS-1313687 and DMS-1614948. MN and BR were partially supported by the University of Pisa via grant PRA-20150017 and by GNAMPA of INdAM. 


\section{Bibliography}

[1] Abramowitz, M.; Stegun, I. A. Handbook of mathematical functions with formulas, graphs, and mathematical tables. National Bureau of Standards Applied Mathematics Series, 55. For sale by the Superintendent of Documents, U.S. Government Printing Office, Washington, D.C., 1964. 3.3 3.2

[2] Alberti, G.; Choksi, R.; Otto, F. Uniform energy distribution for an isoperimetric problem with long-range interactions. J. Amer. Math. Soc. 22 (2009), no. 2, 569-605. doi:10.1090/S08940347-08-00622-X11

[3] Ambrosio, L.; Caselles, V.; Masnou, S.; Morel, J.-M. Connected components of sets of finite perimeter and applications to image processing. J. Eur. Math. Soc. (JEMS) 3 (2001), no. 1, 39-92. doi:10.1007/PL00011302 2] 3.3

[4] Ambrosio, L.; Fusco, N.; Pallara, D. Functions of bounded variation and free discontinuity problems. Oxford Mathematical Monographs. The Clarendon Press, Oxford University Press, New York, 2000.2 3.3

[5] Andelman, D.; Broçhard, F.; Joanny, J.-F. Phase transitions in Langmuir monolayers of polar molecules. J. Chem. Phys. 86 (1987), 3673-3681. doi:10.1063/1.451970 1

[6] Barrero, A.; Loscertales, I. G. Micro- and nanoparticles via capillary flows. Annu. Rev. Fluid Mech. 39 (2007), 89-106. doi:10.1146/annurev.fluid.39.050905.110245[1]

[7] Basaran, O. A.; Scriven, L. E. Axisymmetric shapes and stability of isolated charged drops. Phys. Fluids A. 1 (1989), 795-798. doi:10.1063/1.857551[1]

[8] Bohr, N.; Wheeler, J. A. The mechanism of nuclear fission. Phys. Rev. 56 (1939), no. 5, 426450. doi:10.1103/PhysRev.56.42611

[9] Bonacini, M.; Cristoferi, R. Local and global minimality results for a nonlocal isoperimetric problem on $\mathbb{R}^{N}$. SIAM J. Math. Anal. 46 (2014), no. 4, 2310-2349. doi:10.1137/1309298981

[10] Borell, C. Capacitary inequalities of the Brunn-Minkowski type. Math. Ann. 263 (1983), no. 2 , 179-184. doi:10.1007/BF01456879 3.2 3.2

[11] Braides, A. $\Gamma$-convergence for beginners. Oxford Lecture Series in Mathematics and Its Applications, 22. Oxford University Press, Oxford, 2002. doi:10.1093/acprof:oso/9780198507840.001.0001 1

[12] Bucur, D.; Fragalà, I.; Lamboley, J. Optimal convex shapes for concave functionals. ESAIM Control Optim. Calc. Var. 18 (2012), no. 3, 693-711. doi:10.1051/cocv/2011167/3.2

[13] Burton, J. C.; Taborek, P. Simulations of Coulombic fission of charged inviscid drops. Phys. Rev. Lett. 106 (2011), no. 14, 144501. doi:10.1103/PhysRevLett.106.144501[1]

[14] Caffarelli, L. A.; Jerison, D.; Lieb, E. H. On the case of equality in the Brunn-Minkowski inequality for capacity. Adv. Math. 117 (1996), no. 2, 193-207. doi:10.1006/aima.1996.0008 3.2

[15] Castro-Hernández, E.; Garcia-Sanchez, P.; Tan, S. H.; Ganan-Calvo, A. M.; Baret, J.-C.; Ramos, A. Breakup length of AC electrified jets in a microfluidic flow-focusing junction. $\mathrm{Mi}$ crofluid. Nanofluid. 19 (2015), no. 4, 787-794. doi:10.1007/s10404-015-1603-31

[16] Choksi, R.; Peletier, M. A. Small volume fraction limit of the diblock copolymer problem: I. Sharp-interface functional. SIAM J. Math. Anal. 42 (2010), no. 3, 1334-1370. doi:10.1137/090764888 1]

[17] Choksi, R.; Peletier, M. A. Small volume-fraction limit of the diblock copolymer problem: II. Diffuse-interface functional. SIAM J. Math. Anal. 43 (2011), no. 2, 739-763. doi:10.1137/10079330X 12

[18] Colesanti, A.; Salani, P. The Brunn-Minkowski inequality for $p$-capacity of convex bodies. Math. Ann. 327 (2003), no. 3, 459-479. doi:10.1007/s00208-003-0460-7 3.2 3.2

[19] Di Nezza, E.; Palatucci, G.; Valdinoci, E. Hitchhiker's guide to the fractional Sobolev spaces. Bull. Sci. Math. 136 (2012), no. 5, 521-573. doi:10.1016/j.bulsci.2011.12.004]3.1] 
[20] Dudko, O. K.; Berezhkovskii, A. M.; Weiss, G. H. Rate constant for diffusion-influenced ligand binding to receptors of arbitrary shape on a cell surface. J. Chem. Phys. 121 (2004), no. 3, 15621565. doi:10.1063/1.1763137[3.2] 3.2

[21] Emery, V. J.; Kivelson, S. A. Frustrated electronic phase-separation and high-temperature superconductors. Physica C 209 (1993), no. 4, 597-621. doi:10.1016/0921-4534(93)90581-A [1]

[22] Fernández de la Mora, J. The fluid dynamics of Taylor cones. Annual review of fluid mechanics. Vol. 39, 217-243. Annual Review of Fluid Mechanics, 39. Annual Reviews, Palo Alto, Calif., 2007. doi:10.1146/annurev.fluid.39.050905.110159[1]

[23] Figalli, A.; Fusco, N.; Maggi, F.; Millot, V.; Morini, M. Isoperimetry and stability properties of balls with respect to nonlocal energies. Comm. Math. Phys. 336 (2015), no. 1, 441-507. doi:10.1007/s00220-014-2244-11]

[24] Fontelos, M. A.; Friedman, A. Symmetry-breaking bifurcations of charged drops. Arch. Ration. Mech. Anal. 172 (2004), no. 2, 267-294. doi:10.1007/s00205-003-0298-x 1] 1]

[25] Frank, R. L.; Killip, R.; Nam, P. T. Nonexistence of large nuclei in the liquid drop model. Lett. Math. Phys. 106 (2016), no. 8, 1033-1036. doi:10.1007/s11005-016-0860-8 1]

[26] Frank, R. L.; Lieb, E. H. A compactness lemma and its application to the existence of minimizers for the liquid drop model. SIAM J. Math. Anal. 47 (2015), no. 6, 4436-4450. doi:10.1137/15M1010658 1

[27] Gamow, G. Mass defect curve and nuclear constitution. Proc. Roy. Soc. London A 126 (1930), no. 803, 632-644. 1

[28] Gardner, R. J. The Brunn-Minkowski inequality. Bull. Amer. Math. Soc. (N.S.) 39 (2002), no. 3, 355-405. doi:10.1090/S0273-0979-02-00941-2 [1

[29] Garzon, M.; Gray, L. J.; Sethian, J. A. Numerical simulations of electrostatically driven jets from nonviscous droplets. Phys. Rev. E 89 (2014), no. 3, 033011. doi:10.1103/PhysRevE.89.033011 1

[30] Gaskell, S. J. Electrospray: Principles and practice. J. Mass Spectrom. 32 (1997), 677-688. doi:10.1002/(SICI)1096-9888(199707)32:7<677::AID-JMS536>3.0.CO;2-G 1

[31] Goldman, D.; Muratov, C. B.; Serfaty, S. The $\Gamma$-limit of the two-dimensional OhtaKawasaki energy. I. Droplet density. Arch. Ration. Mech. Anal. 210 (2013), no. 2, 581-613. doi:10.1007/s00205-013-0657-11

[32] Goldman, D.; Muratov, C. B.; Serfaty, S. The $\Gamma$-limit of the two-dimensional Ohta-Kawasaki energy. Droplet arrangement via the renormalized energy. Arch. Ration. Mech. Anal. 212 (2014), no. 2, 445-501. doi:10.1007/s00205-013-0711-z 1

[33] Goldman, M.; Novaga, M.; Ruffini, B. Existence and stability for a non-local isoperimetric model of charged liquid drops. Arch. Ration. Mech. Anal. 217 (2015), no. 1, 1-36. doi:10.1007/s00205-014-0827-9 1, 2. 2

[34] Goldman, M.; Novaga, M.; Ruffini, B. On minimizers of an isoperimetric problem with longrange interactions and convexity constraint. Preprint, 2016. arXiv:1603.01240 [math.AP] 1]

[35] Julin, V. Isoperimetric problem with a Coulomb repulsive term. Indiana Univ. Math. J. 63 (2014), no. 1, 77-89. doi:10.1512/iumj.2014.63.5185[1]

[36] Knüpfer, H.; Muratov, C. B. On an isoperimetric problem with a competing nonlocal term I: The planar case. Comm. Pure Appl. Math. 66 (2013), no. 7, 1129-1162. doi:10.1002/cpa.21451 112

[37] Knüpfer, H.; Muratov, C. B. On an isoperimetric problem with a competing nonlocal term II: The general case. Comm. Pure Appl. Math. 67 (2014), no. 12, 1974-1994. doi:10.1002/cpa.214791

Should the title of [39]

be: A shorter course of theoretical physics?
[38] Knüpfer, H.; Muratov, C. B.; Novaga, M. Low density phases in a uniformly charged liquid. Comm. Math. Phys. 345 (2016), no. 1, 141-183. doi:10.1007/s00220-016-2654-31

[39] Landau, L. D.; Lifshits, E. M. Course of theoretical physics. Pergamon Press, Springer, New York-Heidelberg, 1972. 11 3.2, 39

[40] Landkof, N. S. Foundations of modern potential theory. Die Grundlehren der mathematischen Wissenschaften, 180. Springer, New York-Heidelberg, 1972.1, 1, 1, 2, 3.1, 3.1 
[41] Lu, J.; Otto, F. Nonexistence of a minimizer for Thomas-Fermi-Dirac-von Weizsäcker model. Comm. Pure Appl. Math. 67 (2014), no. 10, 1605-1617. doi:10.1002/cpa.21477[1

[42] Maz'ja, V. G.; Havin, V. P. A nonlinear potential theory. Uspehi Mat. Nauk 27 (1972), no. 6, 67-138. 3.1

[43] Muratov, C. B.; Novaga, M. On well-posedness of variational models of charged drops. Proc. A. 472 (2016), no. 2187, 20150808, 12 pp. doi:10.1098/rspa.2015.0808[1] 2

[44] Muratov, C. B.; Zaleski, A. On an isoperimetric problem with a competing non-local term: Quantitative results. Ann. Global Anal. Geom. 47 (2015), no. 1, 63-80. doi:10.1007/s10455014-9435-z 1] 2

[45] Novaga, M.; Ruffini, B. Brunn-Minkowski inequality for the 1-Riesz capacity and level set convexity for the 1/2-Laplacian. J. Convex Anal. 22 (2015), no. 4, 1125-1134. 1] 2 [3.2

[46] Pólya, G.; Szegö, G. Inequalities for the capacity of a condenser. Amer. J. Math. 67 (1945), 1-32. doi:10.2307/23719121, 4

[47] Pólya, G.; Szegö, G. Isoperimetric inequalities in mathematical physics. Annals of Mathematics Studies, 27. Princeton University Press, Princeton, N.J., 1951.1] 4

[48] Rayleigh, L. On the equilibrium of liquid conducting masses charged with electricity. Phil. Mag. 14 (1882), 184-186. doi:10.1080/14786448208628425[1] []

[49] Samaddar, S.; Yudhistira, I.; Adam, S.; Courtois, H.; Winkelmann, C. B. Charge puddles in graphene near the Dirac point. Phys. Rev. Lett. 116 (2016), no. 12, 126804. doi:10.1103/PhysRevLett.116.126804[1]

[50] Schneider, R. Convex bodies: The Brunn-Minkowski theory. Second expanded edition. Encyclopedia of Mathematics and Its Applications, 151. Cambridge University Press, Cambridge, 2014. 3.2

[51] Taylor, G. Disintegration of water drops in an electric field. Proc. Roy. Soc. Lond. A 280 (1964), no. 1382, 383-397. doi:10.1098/rspa.1964.0151[1]

[52] Weizsäcker, C. F. Zur Theorie der Kernmassen. Zeitschrift für Physik 96 (1935), no. 7-8, 431458. doi:10.1007/BF01337700[1]

[53] Zeleny, J. Instability of elecrtrified liquid surfaces. Phys. Rev. 10 (1917), no. 1, 1-6. doi:10.1103/PhysRev.10.11

Cyrill B. Muratov

Department of Mathematical Sciences

New Jersey Institute of Technology

Newark, NJ 07102

USA

E-mail: muratov@njit.edu

\section{BERARDO RUFFINI}

Institut Montpelliérain

Alexander Grothendieck

Université de Montpellier

Place Eugene Bataillon

34095, Montpellier Cedex 5

FRANCE

E-mail: berardo.ruffini@

umontpellier.fr
Matteo Novaga

Department of Mathematics

University of Pisa

Largo B. Pontecorvo 5

Pisa 56127

ITALY

E-mail: matteo.novaga@unipi.it 